\title{
The study on the antioxidant activity of polysaccharides isolated from Polygonatum odoratum (Mill.) Druce
}

\author{
Wulan Gerile ${ }^{1,2}$, Naranchimeg Dorjpalam ${ }^{1 *}$, Wanyuan Gui², Liang $\mathrm{Xu}^{2}$, Jinglin Liu² \\ ${ }^{1}$ School of Mathematics and Natural Sciences, Mongolian National University of Education; \\ Ulaanbaatar 210648, Mongolia \\ ${ }^{2}$ College of Chemistry and Materials, Inner Mongolia University for the Nationalities, \\ Tongliao 028043, P. R. China \\ *Corresponding author: naranchimeg@msue.edu.mn
}

Received: 13 September 2019; revised: 2 June 2020; accepted: 26 June 2020

\begin{abstract}
The polysaccharides isolated from Polygonatum odoratum (Mill.) Druce (POPs) by water extraction, after precipitation with ethanol were purified through deproteinization, decolorization, dialysis, and column chromatography leading to a purified polysaccharide (POPs-I) content of $90.7 \%$. The scavenging of oxygen free radicals and inhibition of lipid peroxidation (LPO) by POPs-I were analyzed using a colorimetric method. The results showed that the hydroxyl radical scavenging ability of the polysaccharides was weaker than that of benzoic acid, but stronger than those of ascorbic acid and mannitol, and that the superoxide anion radical scavenging ability was inferior to those of all three. When the concentration was higher than $1.0 \mathrm{mg} / \mathrm{mL}$, the POPs-I could inhibit LPO by superoxide anion radicals to a certain degree. Therefore, this work suggests that POPs-I are potential antioxidant agents in medicine and functional food.
\end{abstract}

Keywords: Polygonatum odoratum (Mill.) Druce; Polysaccharide; Antioxidant activity

\section{INTRODUCTION}

Polygonatum odoratum (Mill.) Druce (PO) is an edible medicinal plant that has been recorded in both the Shen Nong Ben Cao Jing and Ben Cao Zheng Yi, two ancient dictionaries of Chinese herbs [1], for hundreds of years as a typical Chinese herb. Currently, it is recorded in the pharmacopeia of the People's Republic of China $[2,3]$. In recent years, a series of research studies have reported the anti-aging effects of Polygonatum odoratum [4], antidiabetic effects of total saponins from Polygonatum odoratum [5], and effects of this planton hypoxia tolerance in mice [6]. There is increasing evidence indicating that Polygonatum odoratum contains a variety of ingredients such as steroidal saponins, flavonoids, alkaloids, polysaccharides, and cardiac glycosides. The polysaccharide is one of the main active ingredients of Polygonatum odoratum [7-9] and its physiological activity includes lowering blood pressure and blood fat and improving myocardial ischemia [10]. To date, there have been many reports on extraction, separation, purification, structure and pharmacology of polysaccharides from Polygonatum odoratum. Yong Chen et al. [11] reported the optimization of alkaline extraction and bioactivities of polysaccharides from rhizome of Polygonatum odoratum, and several other reports were documented on extraction and bioactivities of polysaccharides from Polygonatum odoratum in recent years. For example, Lan et al. applied response surface methodology to optimize the conditions for ultrasound-assisted extraction of polysaccharides from Polygonatum odoratum polysaccharides [12], and Jiang et al. reported optimal extraction parameters and liver protective effect of the polygonatum polysaccharides in vivo [13]. Furthermore, Zong [14], Li [15], Wang [16], Bu et al. [17] reported different production areas, varieties, growth years, extraction and separation methods and analysis conditions will affect the content, composition and biological activity of Polygonatum odoratum polysaccharides.

In this study, the dried rhizomes of the biennial Polygonatum odoratum (Mill.) Druce (PO) made in Zhejiang Province were used as material, polysaccharides (POPs) were extracted with hot water and precipitated using ethanol, and then purified through decolorization, and dialysis, 
followed by column chromatography. Finally, the antioxidant activities of the polysaccharides (POPs-I) from $\mathrm{PO}$ were investigated.

\section{EXPERIMENTAL}

Materials and reagents: Instruments used were as follows: chromatography column $(100 \mathrm{~cm} \times 5 \mathrm{~cm})$, UV-240 Spectrophotometer (Shimadzu Corporation, Japan), NICOLET 5700 FT-IR infrared spectrometer (PerkinElmer, USA), 722s visible spectrophotometer (Shanghai Precision Scientific Instrument Co. Ltd.) dialysis bag (Beijing Dingguo Changsheng Biotechnology Co. Ltd., import sub pack, 36 DM), Highspeed centrifuge (Shanghai Anting Scientific Instrument Co. Ltd., TDL-5).

POD was purchased from Tongliao, Inner Mongolia Province, China, and authenticated by Professor Wang F, Inner Mongolia University for the Nationalities, Inner Mongolia, China. The rhizome pieces were ground to a powder with a grinding machine and sieved with a 60-mesh screen then stored at $60^{\circ} \mathrm{C}$ in a drier. DEAECellulose, Sephadex G-100, thiobarbituric acid (TBA), nitro blue tetrazolium (NBT), and tris-(hydroxymethyl)amino methane were purchased from Sigma Chemical Co. (St. Louis, MO, USA).

Safranine T, benzoic acid, mannitol, and ascorbic acid (Vc) were purchased from Shantou Guanghua Chemical Co. (Guangdong, China). Absolute ethanol (ethanol mass fraction $\geq 99.9 \%$ ) and other reagents were of analytical grade. Ultra-pure water was used throughout the experiments. Clean-grade Kunming mice weighing $(20 \pm 2) \mathrm{g}$ were provided by the Inner Mongolia University for Nationalities Experimental Animal Center (Certificate of Conformity: SCXK (Jilin) 2007-0003).

Extraction of polysaccharides and determination of yield: The raw PO was ground into a fine powder using a disintegrator. Using a water bath, $20.0 \mathrm{~g}$ of PO powder was refluxed three times in $200 \mathrm{~mL}$ of water at $80^{\circ} \mathrm{C}$ for $3 \mathrm{~h}$. After filtration, the combined filtrates were evaporated and concentrated under reduced pressure to a volume of $45 \sim 50 \mathrm{~mL}$, and then cooled to room temperature. $100 \mathrm{~mL}$ of absolute ethanol was added, and precipitation was allowed to occur for $24 \mathrm{~h}$. The precipitate was filtered off, washed sequentially with absolute ethanol and acetone, and finally, lyophilization is carried out to obtain crude POPs. Afterward, the polysaccharide content was measured using the phenol-sulfuric acid method with D-glucose as the standard [18].

Qualitative analysis of POPs: Qualitative analysis of POPs was conducted from the following aspects. Molisch reaction: At first, we carried out Molisch's test for the identification of carbohydrates. A small amount $(1.0 \mathrm{~mL})$ of a $1.0 \mathrm{~mol} / \mathrm{L}$ solution of POPs was placed in a test tube, and then two drops of Molisch's reagent and $1.0 \mathrm{~mL}$ of concentrated sulfuric acid were added in turn. After the mixture was allowed to stand for several minutes, a purple color was observed at the interface of the two surfaces, indicating that the solution contained polysaccharides.

Ninhydrin reaction: The Ninhydrin test was used to detect traces of any protein in POPs. A small amount $(1.0 \mathrm{~mL})$ of a $1.0 \mathrm{~mol} / \mathrm{L}$ solution of POPs was placed in a test tube followed by $0.5 \mathrm{~mL}$ of a ninhydrin solution. The mixture was heated at $100{ }^{\circ} \mathrm{C}$ in a water bath for $10 \mathrm{~min}$. No changes were observed in the solution, indicating that it did not contain proteins.

lodine-potassium iodide reaction: The lodine test was used to examine the presence of starch in a solution of crude POPs. A small amount $(1.0 \mathrm{~mL})$ of a $1.0 \mathrm{~mol} / \mathrm{L}$ solution of POPs was placed in a test tube followed by two drops of iodine-potassium iodide reagent. After the mixture was shaken, no significant changes were observed, indicating that starch was not present.

Purification of POPs: $\mathrm{H}_{2} \mathrm{O}_{2}$ bleaching: $2 \mathrm{~g}$ of POPs were dissolved in $400 \mathrm{~mL}$ of distilled water in a large beaker. The $\mathrm{pH}$ of the POP solution was adjusted to $8 \sim 9$ with ammonia, and then one-tenth the volume of a $15 \% \mathrm{H}_{2} \mathrm{O}_{2}$ solution was added and the resulting solution was heated at $50{ }^{\circ} \mathrm{C}$ in a water bath for $1 \mathrm{~h}$. After the solution had cooled to room temperature for $24 \mathrm{~h}$, the resulting precipitate was collected by filtration and the filtrate was concentrated under reduced pressure. After precipitation with absolute ethanol, filtration, washing, and drying, white powdery POPs were obtained.

Desalting and dialysis: The bleached POPs were dissolved in distilled water and placed in dialysis bags, and then dialysis was conducted with flowing water for $12 \mathrm{~h}$. The dialysate underwent a series of processes including concentration, precipitation with ethanol, filtration, washing, and drying to afford dried and crude POPs.

Purification by column chromatography: Column chromatography was conducted according to previously reported methods $[19,20]$. The above crude POPs were dissolved in deionized water. A column with the DEAE Cellulose was balanced with two volumes of a $0.1 \mathrm{~mol} / \mathrm{L}$ $\mathrm{NaCl}$ solution as the eluent and deionized water at a flow rate of $0.5 \mathrm{~mL} / \mathrm{min}$. The POPs solution was loaded carefully onto the above gel column and eluted with a $0.1 \mathrm{~mol} / \mathrm{L} \mathrm{NaCl}$ solution at a flow rate of $0.5 \mathrm{~mL} / \mathrm{min}$ and collected automatically in $2 \mathrm{~mL}$ tubes. The eluate was monitored using the phenol-sulfuric acid method. The main fraction was collected and concentrated. After precipitation with ethanol, washing, and drying, white POPs-I powder was obtained. The elution curve is shown in Figure 1. Further purification was conducted by eluting with deionized water on a column of Sephadex G-100 molecular sieve chromatography. The eluate was collected and lyophilized, affording the purified POPs-I. The elution curve is shown in Figure 2. Infrared and ultraviolet spectroscopy assays of POPs-I: Infrared spectroscopy of POPs: The test sample was prepared using the potassium bromide 
tablet method and scanned in the $400 \sim 4000 \mathrm{~cm}^{-1}$ range. The infrared (IR) absorption spectrum of the POPs-I is shown in Figure 3.

Ultraviolet spectroscopy of POPs-I: The POPs-I were dissolved in distilled water and the ultraviolet (UV) absorption spectrum in the $200 \sim 400 \mathrm{~nm}$ range was obtained. The obtained UV spectrum of the POPs-I is shown in Figure 4.

Lipid peroxidation inhibition and removal of oxygen free radicals by POPs-I: Preparation of test solution and standard solutions: The POPs-I, ascorbic acid (Vc), mannitol, and benzoic acid were dissolved in deionized water, and these solutions were diluted to various concentrations as required before use. They were $0.28,0.64,1.00,1.36,1.82$, and $2.08 \mathrm{mg} / \mathrm{mL}$ for the POPs-I, Vc, and mannitol standard solutions and $0.32,0.50,0.68,0.86$, and $1.04 \mathrm{mg} / \mathrm{mL}$ for the benzoic acid standard solution.

Assay for hydroxyl radical scavenging activity: This assay was conducted according to the method reported by Cheng et al. [21]. $\mathrm{OH}$ was generated from an ethylenediaminetetraacetate (EDTA)-2 $\mathrm{Na}-\mathrm{Fe}(\mathrm{II})-\mathrm{H}_{2} \mathrm{O}_{2}$ system. Since $\cdot \mathrm{OH}$ can cause safranine $\mathrm{T}$ to fade, $\cdot \mathrm{OH}$ content can be measured according to the degree of fading. The reaction system was a mixture of $1.5 \mathrm{~mL}$ of a $0.05 \mathrm{~mol} / \mathrm{L}$ sodium phosphate buffer $(\mathrm{pH}=7.4), 0.2$ $\mathrm{mL}$ of a $520 \mu \mathrm{g} / \mathrm{mL}$ safranine $\mathrm{T}$ solution, $0.7 \mathrm{~mL}$ of a $2.0 \mathrm{mmol} / \mathrm{L}$ EDTA-2Na-Fe(II) solution, and $0.4 \mathrm{~mL}$ of a $6 \% \mathrm{H}_{2} \mathrm{O}_{2}$ solution. After $1.0 \mathrm{~mL}$ of the sample solution was added, the mixture was incubated at $37^{\circ} \mathrm{C}$ for $1 \mathrm{~h}$. The absorbance at $510 \mathrm{~nm}$ was measured. Deionized water was used as blank control. Benzoic acid [22], Vc $[22,23]$ which are substances with strong antioxidant effects and mannitol $[22,24]$ which is polyhydric alcohol with a certain degree of reducibility, are used as positive controls. The $\cdot \mathrm{OH}$ scavenging activity was calculated using the following equation:

$$
\text { Scavenging activity }(\%)=\frac{A_{1}-A_{2}}{A_{3}-A_{2}} \times 100 \%
$$

where $A_{1}$ is the absorbance of the test sample mixed with the reaction solution, $A_{2}$ is the absorbance when the sample was replaced with deionized water, and $\mathrm{A}_{3}$ is the absorbance when $\mathrm{H}_{2} \mathrm{O}_{2}$ and the sample were replaced with deionized water.

Assay for superoxide anion radical scavenging activity: Superoxide anion radicals were generated using the pyrogallol autoxidation system under alkalescent conditions. The method for measuring superoxide anion radical $\left(\cdot \mathrm{O}_{2}^{-}\right)$scavenging activity was described in a previous report [18]. In a test tube was placed 2.5 $\mathrm{mL}$ of a $0.1 \mathrm{~mol} / \mathrm{L}$ Tris- $\mathrm{HCl}$ buffer $(\mathrm{pH}=8.2)$, which was preheated at $25^{\circ} \mathrm{C}$ in a water bath for $20 \mathrm{~min}$. Then, $0.2 \mathrm{~mL}$ of sample solution with different concentrations was added, followed by $0.6 \mathrm{~mL}$ of a $0.98 \mathrm{mmol} / \mathrm{L}$ NBT solution and $0.3 \mathrm{~mL}$ of a $10 \mathrm{mmol} / \mathrm{L}$ pyrogallol solution. The reaction proceeded in a water bath at $25^{\circ} \mathrm{C}$ for
$4 \mathrm{~min}$. Then, $0.1 \mathrm{~mL}$ of an $8 \mathrm{~mol} / \mathrm{L}$ hydrochloric acid solution was used to terminate the reaction. The absorbance at $530 \mathrm{~nm}$ was measured. Benzoic acid, $\mathrm{Vc}$, and mannitol were used as reference materials. The superoxide anion radical scavenging activity was calculated using the following equation:

$$
\text { Scavenging activity }(\%)=\frac{A_{B}-A_{s}}{A_{B}} \times 100 \%
$$

where $A s$ is the absorbance of the sample and $A_{B}$ is the absorbance when the sample was replaced with distilled water.

Assay for lipid peroxidation inhibition activity:

In the lipid peroxidation (LPO) reaction, $\cdot \mathrm{OH}$ reacts with unsaturated fatty acids in liver homogenates and the final product is malondialdehyde (MDA). In the presence of acetic acid, the reaction between TBA and MDA results in coloration, and the relative concentration of MDA can be calculated from the degree of coloration. The reaction system contained $0.5 \mathrm{~mL}$ of a $10 \%$ liver homogenate solution [18], $1.0 \mathrm{~mL}$ of a phosphate buffer ( $\mathrm{pH}=7.4), 2.0 \mathrm{~mL}$ of a $2 \mathrm{mmol} / \mathrm{L}$ EDTA-2Na$\mathrm{Fe}$ (II) solution, $1.0 \mathrm{~mL}$ of a $3 \% \mathrm{H}_{2} \mathrm{O}_{2}$ solution, and 10 $\mathrm{mL}$ of sample solutions with different concentrations. Each mixture was mixed uniformly and heated in a water bath at $37^{\circ} \mathrm{C}$ for $30 \mathrm{~min}$ and then cooled to room temperature. A small amount of the mixture $(0.3 \mathrm{~mL})$ was drawn and placed in a small test tube, and then $0.6 \mathrm{~mL}$ of $5 \%$ TBA and $1.2 \mathrm{~mL}$ of $20 \%$ acetic acid were added. The tubes were heated in a water bath at $95^{\circ} \mathrm{C}$ for $1 \mathrm{~h}$ and then cooled to $25^{\circ} \mathrm{C}$. The absorbance at 530 $\mathrm{nm}$ was measured. An equal volume of distilled water instead of test solution was used as a blank control.

The LPO inhibition activity was calculated using the following equation:

$$
\text { Scavenging activity (\%) }=\frac{A_{0}-A_{s}}{A_{0}} \times 100 \%
$$

where $A_{s}$ is the absorbance of the sample and $A_{0}$ is the absorbance of the blank control (distilled water instead of samples).

\section{RESULTS AND DISCUSSION}

Polysaccharide concentration: The crude polysaccharide (POPs) content was measured using the phenol-sulfuric acid method to be $54.6 \%$, the purified POPs-I content was $90.7 \%$. Using glucose as the standard, the following regression equation was obtained: $y=8.2355 x-0.0216\left(R^{2}=0.9976, y\right.$ is the absorbance and $x$ is the concentration). This was similar to the result obtained by Xie et al. [25] that the average content of polysaccharides extracted from Polygonatum odoratum under the optimal conditions was $86.02 \%$ by orthogonal test. 
Purification polysaccharide: At present, the main method to separate and purify the polysaccharides is column chromatography, and there are also methods using ethanol fractional precipitation or quaternary ammonium salt precipitation [14]. Sun Ping et al. [27] used semi-permeable membrane and Sephadex G-75 gel column chromatography to separate the watersoluble polysaccharides of Polygonatum odoratum. Wang Qiang et al. [26] isolated and purified neutral polysaccharide from Polygonatum odoratum by DEAE-cellulose ion-exchange chromatography and Sepharose CL-6B molecular sieve chromatography. In this paper, the crude polysaccharide was purified through decolorization, dialysis, then using the DEAECellulose column chromatography to separate POPs-I, and eluted with $0.1 \mathrm{~mol} / \mathrm{L} \mathrm{NaCl}$ solution. The elution curve is shown in Figure.1.

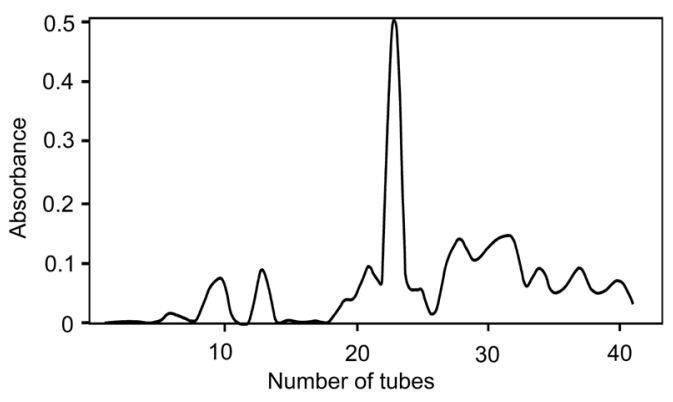

Fig. 1. DEAE-cellulose column chromatogram of POPs.

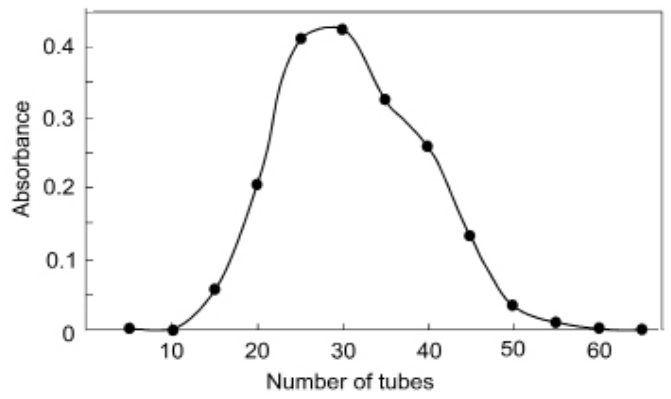

Fig. 2. Sephadex G-100 molecular sieve column chromatogram of POPs-I.

Further purification was conducted by eluting with deionized water on a column of Sephadex G-100 molecular sieve chromatography. The elution curve is shown in Figure 2.

IR and UV spectra of the POPs: Fourier transform infrared (FT-IR) spectroscopy and UV spectroscopy were used to investigate the functional group structure and purity of the purified polysaccharides.

Infrared spectral analysis: The IR spectrum of the POPs-I is shown in Figure 3 . The broad bands at $3440.1 \mathrm{~cm}^{-1}$ were assigned to the $\mathrm{v}(\mathrm{O}-\mathrm{H})$ stretching vibrations of hydrogen-bonded and free hydroxyl groups [28], and the signal at $2921.7 \mathrm{~cm}^{-1}$ was attributed to $\mathrm{v}(\mathrm{C}-\mathrm{H})$ stretching of $\mathrm{CH}_{2}$ groups [14,
29], at $1631.8 \mathrm{~cm}^{-1}$ corresponded to the absorption of the carboxylate anions was $(\mathrm{O}=\mathrm{C}-\mathrm{O})$ of nonesterified groups in pectins [28], and near the signal at 1378.8 $\mathrm{cm}^{-1}$ was COO- symmetric stretching vibration.

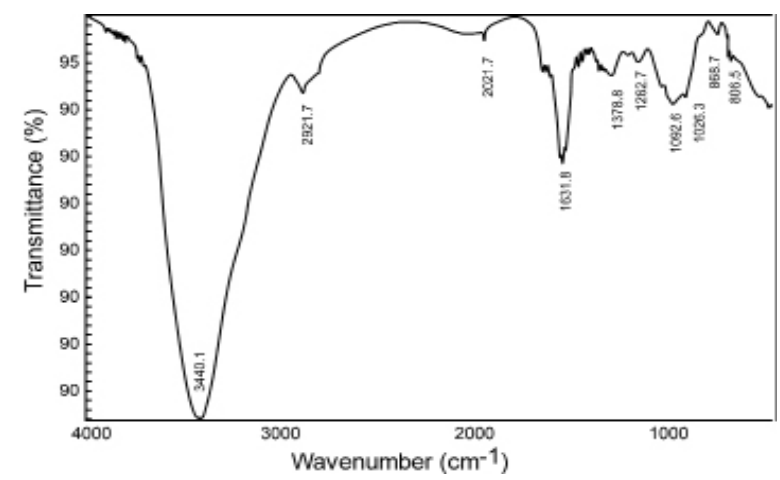

Fig. 3. IR spectrum of the POPs-I.

The peak at $1262.7 \mathrm{~cm}^{-1}$ was $\mathrm{v}(\mathrm{C}-\mathrm{O})$, and the absorption bands analysis of the spectrum in the $1092.6 \mathrm{~cm}^{-1}, 1026.3 \mathrm{~cm}^{-1}$ regions showed specific bands typical for a pyranose ring vibration [28, 29]. The peak at $868.7 \mathrm{~cm}^{-1}$ indicated the presence of $\beta$-glycosidic linkage in the molecular structure of the polysaccharides [14, 28].

UV assay: In the UV spectrum (Figure 4), there are no significant absorption peaks at 260 and $280 \mathrm{~nm}$, indicating that no nucleic acids or proteins were present in the purified POPs-I [11], which is consistent with the results of the qualitative analysis.

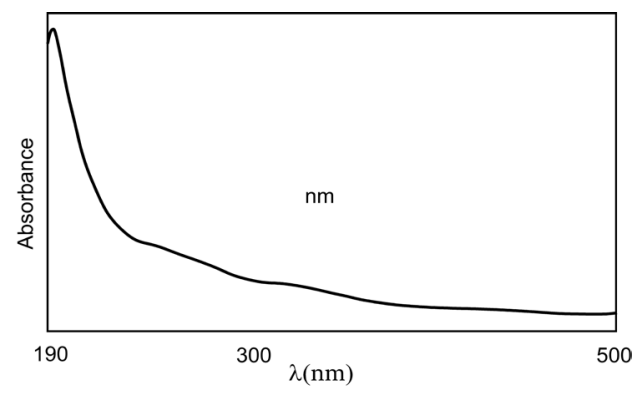

Fig. 4. UV spectrum of the POPs-I.

Removal of oxygen free radicals and lipid peroxidation inhibition by the polysaccharides:

- $\mathrm{OH}$ scavenging ability of the POPs-I:

Benzoic acid, Vc, and mannitol were used as standard materials, and the scavenging ability of the POPs of hydroxyl free radicals was evaluated.

The results are shown in Figure 5. The figure shows that the scavenging abilities of the samples and standard materials were dose dependent.

The scavenging ability of the POPs-I of hydroxyl free radicals was inferior to that of benzoic acid but superior to those of $\mathrm{Vc}$ and mannitol. Zong, et al. [14] reported that the scavenging capacity for hydroxyl radicals of Polysaccharides (POA, POA-70S, POA-70P) separated from Polygonatum by ethanol 
fractionation precipitation method was apparently lower than ascorbic acid ( $\mathrm{Vc}$ ). Chen et al. extracted the alkaline soluble polysaccharides from Polygonatum odoratum, which scavenging capacity was also lower than ascorbic acid, it was indicated that during the processing method, $\mathrm{NaOH}$ might change the chemical structure of polysaccharides [11].

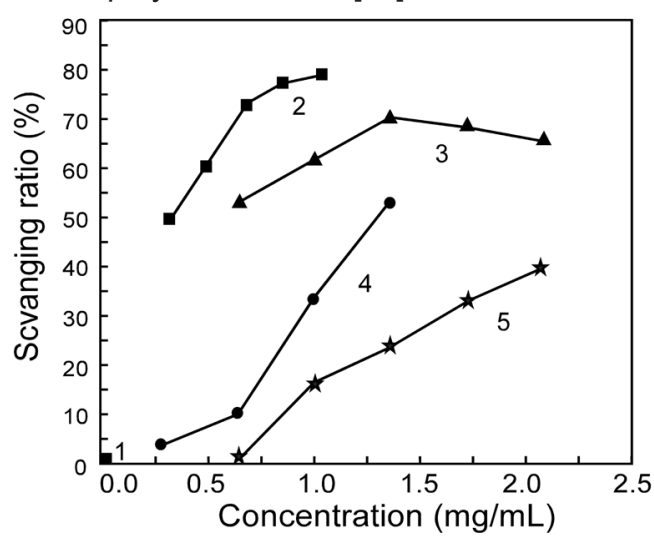

Fig. 5. UV spectrum of the POPs-I

Superoxide anion radical scavenging activity of the POPs-I: Benzoic acid, Vc, and mannitol were used as positive controls, and the results obtained for the $\cdot \mathrm{O}_{2}$ - scavenging activities of the polysaccharides and reference materials are shown in Figure 6.

Figure 6 shows that the $\cdot \mathrm{O}_{2}$ - scavenging ability of the POPs-I was inferior to those of the three reference materials. The scavenging abilities of the POPs-I and reference materials were dose dependent.

Lipid peroxidation inhibition by the POPs-I: MDA is one of the end products of the unsaturated fatty acid peroxidation in phospholipids, and has been widely used as a marker of oxidative stress [12, 30].

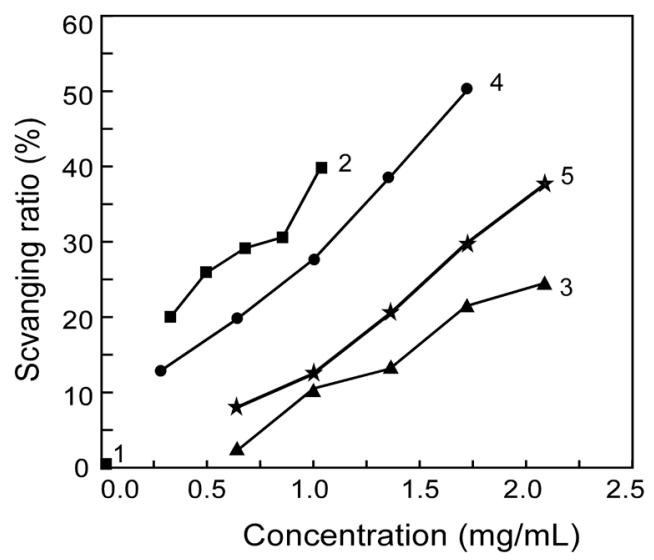

Fig. 5. $\cdot \mathrm{O}_{2}$ - scavenging capacity: 1 - blank solution; 2 - benzoic acid; 3 - Vc; 4 - mannitol; 5 - POPs-I

The data listed in Table 1 show that there is no LPO inhibition effect at low POPs-I concentrations. However, when the concentration was higher than $1.0 \mathrm{mg} / \mathrm{mL}$, the POPs-I showed some LPO inhibition ability, which was dose dependent. This was similar to research results of Lan et al. [12].
Table 1. Lipid peroxidation inhibition ability of the POPs-I $(n=5)$

\begin{tabular}{ccc}
\hline Testing solution & $\begin{array}{c}\text { Concentration } \\
(\mathbf{m g} / \mathrm{mL})\end{array}$ & $\begin{array}{c}\text { Clearance rate } \\
\mathrm{E} \%(\mathbf{X} \pm \mathbf{S})\end{array}$ \\
\hline & 0.64 & - \\
POPs-I & 1.00 & - \\
& 1.36 & $1.14 \pm 0.2$ \\
& 1.72 & $19.32 \pm 0.1$ \\
& 2.08 & $34.09 \pm 0.3$ \\
\hline
\end{tabular}

\section{CONCLUSION}

The results showed that the POPs-I had different scavenging abilities for $\cdot \mathrm{OH}$ and $\mathrm{O}_{2}^{-}$. At equal concentrations, the scavenging ability of the POPs-I for $\cdot \mathrm{OH}$ was superior to that for $\mathrm{O}_{2}^{-}$. At low concentrations, the POPs-I showed no inhibition activity in the production of MDA through lipid peroxidation. When the concentration was higher than $1.0 \mathrm{mg} / \mathrm{mL}$, the POPs-I showed some LPO inhibition. The results indicated that the POPs exhibit concentration-dependent antioxidant activities and suggested that Polygonatum odoratum (Mill.) Druce extracts are potential suitable natural antioxidants and functional medicines for the treatments of certain diseases.

\section{ACKNOWLEDGMENTS}

This study was supported by the National Natural Science Foundation of China $(21362020,81560702)$, the Natural Science Foundation of Inner Mongolia Autonomous Region, China (2015MS0220)

\section{REFERENCES}

1. Huang Z.S. (2002) Chinese Pharmacy, People's Health Publishing House, Beijing, 462. (in Chinese)

2. Pharmacopoeia Commission of People's Republic of China, People's Republic of China Pharmacopoeia: A, Chinese Medical Science and Technology Press, Beijing, 2010, 78. (in Chinese)

3. Zhou Y., Tang C., Gao X., Wang R.L. (2005) Research progress on traditional Chinese medicine Polygonatum odoratum (Mill.) Druce. Journal of Tianjin Medical University, 11, 328-330. (in Chinese)

4. Wang Y.Q., Chen Q.Y., Qi Y.H., Ren Y.D., Zhang N. (2010) Research progress on anti-aging effect of traditional Chinese medicine Polygonatum odoratum. Chinese Journal of Ethnomedicine and Ethnopharmacy, 14, 12-13. (in Chinese)

5. Guo Ch.R., Dai P., Zhang X., Yang Z.L. (2011) Experimental study on antidiabetic effect of total saponins from Polygonatum odoratum. Strait Pharmaceutical Journal, 23, 19-23. (in Chinese)

6. Sun L.Y., Liu Z.L., Sun J.X., Gao Y.S. (2008) Zhao Y.T. Effect of polygonatum odoratum polysaccharides on hypoxia tolerance in mice. Journal of Shandong Agricultural University (Natural Science), 39, 335-338. (in Chinese) 
7. Li H.M., Bai H., Li W., Wang Y.S., Zhao H.X. (2010) Study on chemical constituents of Polygonatum odoratum (Mill.) Druce. Food and Drug, 12, 102104. (in Chinese)

8. Song A.H., Lin Q.S., Zhao C.J. (2013) Effects of three extraction methods on volatile chemical constituents of Polygonatum odoratum (Mill.) Druce by GC-MS. J. Shenyang Pharm. University, 30, 944-947. (in Chinese)

9. Liu T.S., Yang X.G., Gong L.M. (2008) Research progress of medicinal and edible traditional medicine Polygonatum odoratum. Cent. South Pharm., 6, 216-219. (in Chinese)

10. Huang S. Q., Ding S. D., Fan L.P. (2012) Antioxidant activities of five polysaccharides from Inonotus obliquus. Int. J. Biol. Macromol., 50, 1183-1187. https://doi.org/10.1016/j.ijbiomac.2012.03.019

11. Chen Y., Yin L., Zhang X., Wang Y., Chen Q., Min C. et al. (2014) Optimization of alkaline extraction and bioactivities of polysaccharides from rhizome of Polygonatum odoratum. Biomed Res., Int., 2014, 504896. https://doi.org/10.1155/2014/504896

12. Lan G., Chen H., Wang Z., Zhang W., and Zhang L. (2011) Extraction of Polygonatum odoratum polysaccharides using response surface methodology and preparation of a compound beverage. Carbohydrate Polymers, 86, 1175-1180. https://doi.org/10.1016/j.carbpol.2011.06.009

13. Jiang Q., Lv Y., Dai W., Miao X., and Zhong D. (2013) Extraction and bioactivity of Polygonatum polysaccharides. Int. J. Biol. Macromol., 54, 131135. https://doi.org/10.1016/j.ijbiomac.2012.12.010

14. Zong X.Y. (2019) Isolation, purification and structural characterization of polysaccharides from Polygonatum odoratum. Nanchang University, (in Chinese)

15. Li M., Xi G.S., Luo Y.Y., Zou L.L. (2015) Study on the antioxidant activity of polysaccharides and flavonoids in different strains of Polygonatum odoratum. Northern Horticulture, 83, 135-138. (in Chinese)

16. Wang D.M., Lv Z.J., Wang Y.H., Zhang H.H. (2012) Effect of different processing methods on the yield of extracts and antioxidant activities of Polygonatum odoratum. Bull. Bot. Res., 32, 621-626. https://doi. org/10.7525/j.issn.1673-5102.2012.05.020

17. Bu J., Li D.W., Wang D.M. (2012) Correlations between wild Polygonatum odoratum quality and main ecological factors. Chinese Journal of Applied Ecology, Jun. 23, 1447-1454. (in Chinese)

18. Ye C.L., Hu W.L., Dai D.H. (2011) Extraction of polysaccharides and the antioxidant activity from the seeds of Plantago asiatica L. Int. J. Biol. Macromol., 49, 466-470. https://doi.org/10.1016/j.ijbiomac.2011.05.026
19. Li Z., Liu M., He Z.X. (2013) Isolation, purification and monosaccharide analysis of an acidic polysaccharide from Polygonatum odoratum. Chin. J. Exp. Tradit. Med. Formulae, 19, 69-72. (in Chinese)

20. Wu L., Xu L., Chi Q., Ma Z.G., Zhang J.M., Ba H.S. (2013) Isolation and purification of polysaccharides from Mongolian medicine Narenmandula and its antioxidant activity. Chin. J. Pharm. Anal., 33, 1285-1288. (in Chinese)

21. Cheng H.R., Feng S.L., Jia X.J., Li Q.Q., Zhou Y.H., Ding C.B. (2013) Structural characterization and antioxidant activities of polysaccharides extracted from Epimedium acuminatum. Carbohydrate Polymers, 92, 63-68. https://doi.org/10.1016/j. carbpol.2012.09.051

22. Li P., Wang Y.H., Ma R.Y. (2003) Study on physiochemical characteristic and antioxidation activity of polysaccharide extracted with sodium hydroxide from fruit of Cornus officinalis. Chinese Traditional and Herbal Drugs, 34, 973-976. (in Chinese)

23. Chen Y., Sun Y.J., Fang W. (2008) Study on antioxidant activity of Clematis chinensis Osbeck polysaccharide. China Journal of Traditional Chinese Medicine and Pharmacy, 23, 266-270. (in Chinese)

24. Jin J., Bian X., Ge P., Jing H., Ding L., Ding D. (2001) The effects of the polysaccharides from dermatocarpo miniatum on oxygen radicals and lipid peroxidation. J. Chin. Med. Mater., 24, 660661. (in Chinese)

25. Xie J.J., Hu M.J., Sun G.J. (2009) Optimization of the extraction process of Polygonatum polysaccharide. Jiangsu Journal of Traditional Chinese Medicine, 41, 59-60. (in Chinese)

26. Wang Q., Li S. Y., Yang F., Liu C.M. (2010) Isolation, purification and monosaccharide analysis of a neutral polysaccharide from Polygonatum odoratum (Mill) Druce. Food Science, 12, 100-103. (in Chinese)

27. Sun P., Wang H.B., Chen Y.Y. (2010) Study on monosaccharide composition of water-soluble polysaccharide POPS-B1 from Polygonatum odoratum. China Food Additives, 31, 166-168, 208. (in Chinese)

28. Ognyanov M., Georgiev Y., Petkova N., Ivanov I., Vasileva I., and Kratchanova M (2018) Isolation and characterization of pectic polysaccharide fraction from in vitro suspension culture of Fumaria officinalis L. International Journal of Polymer Science, 2018, Article ID 5705036, 13. https://doi.org/10.1155/2018/5705036 
29. Gao X., Li X.F., Mu J.J., Chi-Tang Ho, Sua J.Y., et al. (2020) Preparation, physicochemical characterization and anti-proliferation of selenium nanoparticles stabilized by Polyporus umbellatus polysaccharide. Int. J. Biol. Macromol., 152, 605615. https://doi.org/10.1016/j.ijbiomac.2020.02.199
30. Dubois M., Gilles K.A., Hamilton J.K., Rebers P.A., Smith F. (1956) Colorimetric method for determination of sugars and related substances. Analytical Chemistry, 28, 350-356. https://doi.org/10.1021/ac60111a017 